Int. J. of The Soc. of Mat. Eng. for Resources Vol. 2 No. $1 \quad 78 \sim 85$ (1994)

\title{
Original
}

\section{Dynamic Stability of Coal Water Mixtures}

\author{
by \\ ${\text { Takashi } \mathrm{SAEKI}^{\dagger} \text {, Hiromoto UsUI }}^{\dagger}$ and Masazou OGAWA ${ }^{\dagger \dagger}$
}

\begin{abstract}
Dynamic stability and rheological characteristics of highly loaded coal water mixtures (CWM) with stabilizing additives were measured in aid of selecting suitable additives. Five stabilizing additives were tested. The experimental results show that some kinds of polysaccharides are effective to improve the stability of CWMs. These stabilizing additives have long sensible molecular chains and show high elasticity.
\end{abstract}

Key Words : coal water mixture, stabilizing additive,natural polysaccharide, viscoelasticity

\section{Introduction}

The preparation techniques for highly loaded coal water mixtures (CWM) have been highly developed. In order to achieve a good fluidity of CWMs, a suitable dispersant must be added. One dispersant widely used was the condensation product of formalin and naphthalene sulfonic acid (NSF). Recently, more effective dispersants have been reported $^{1}$, e.g., polystyrene sulfonic acid (PSS) and polymethacrylate (PMA). These dispersants can produce more concentrated slurries compared to NSF. However, some CWMs with the addition of a dispersant only show the rapid sedimention of coal particles. This sedimentation phenomenon would cause many troubles in the practical use of CWMs. Furthermore, especially in Japan, most CWMs are imported by CWM tankers and are stored in tanks for several months. Under these conditions, the techniques for the improvement of CWM stability have become more important. Therefore, suitable stabilizing additives must be added to prevent the sedimentation. Fogler ${ }^{2}$ examined the mechanism of stabilization of CWM. In his study, polystyrene latex was chosen as the model system for CWM, and the stability factor was measured by changing $\mathrm{pH}$. He concluded that it was important to find the substance which were responsible for the particle bonding. Sparks et al. ${ }^{3}$ suggested that attapulgite clay was effective for CWM stability. Takao et al. ${ }^{4}$ investigated the effectiveness of CMC (carboxymethyl cellulose), natural polysaccharide and clay (Attapulgite) and concluded that three additives used in their study were all effective. Goethite $(\mathrm{FeOOH})$ which is a fine needlelike particle has been reported to be a

\footnotetext{
Received December 10, 1993

† Department of Chemical Engineering, Yamaguchi University, 2557 Ube, Yamaguchi 755, Japan

$\dagger+$ Food, Food Additive \& Chemical Division, Dainippon Pharmaceutical CO., LTD., 33-94 Enokicho, Suita, Osaka 564, Japan
} 
good stabilizer for CWM under static conditions. These stabilizing additives may produce an interparticle structure in the CWM which prevents the sedimentation of coal particles.

Xanthan gum supplied by Kelco Division of Merck \& Co.,Inc. (brand name: kelzan-D), which is one of polysaccharide additives, shows a significant improvement of the stability of CWMs, but on the other hand, the addition of kelzan - D to CWMs causes a significant increase in viscosity ${ }^{4,5}$. In addition, the prise of xanthan gum is a little expensive. Thus, kelzan-D is not used widely as a stabilizing additives for CWMs.

Recently, some bio - polysaccharides have become available. These are S-194 and S-130 supplied by Dainihon Pharmaceutical Co.,Ltd. They are expected to improve the stability of CWMs because of their molecular structure. It is reported that they have long molecular chains and sensible ionic functions ${ }^{6,7,8}$. Long molecular chains of stabilizing additives may build up an interparticle structure of coal particles. In this study, the effectiveness of these additives were measured in terms of dynamic stability and rheological characteristics. The object of this study is to evaluate the effectiveness of these additives, and in doing so, to obtain a more detailed knowledge of formation of the network structure in the CWM.

\section{Experimental}

\section{1 Preparation of CWM}

Yanzhou coal were used in this study. This coal is a bituminous coal and contains $8.9 \%$ ash. PMA (polymethacrylate) was used as the dispersant. The coal concentration was $68.9 \%$. Stabilizing additives tested in this study are listed in Table 1.

\section{2 Dynamic stability test for CWMs}

The dynamic stability tests were conducted with the use of a transportation simulator. The vibration conditions were set as follows according to the discussion given by Usui et al ${ }^{9}$. Horizontal and sinusoidal vibrations with a maximum acceleration of $1.89 \mathrm{G}$ were applied for 48 hours. Each test CWM was contained in a glass cylinder (Fig.1) having an inner diameter of $36 \mathrm{~mm}$ and a height of $180 \mathrm{~mm}$ and set on a transportation simulator (Fig.2). After a dynamic stability test was performed, a rod penetration test was conducted to measure the depth of hard pack.

\section{3 Rheological measurement for CWMs}

The rheological characteristics of CWMs were measured by means of a coaxial rotating rheo-

Table 1 Stabilizing additives.

$<$ carboxymethyl cellulose $>$

\begin{tabular}{|c|c|c|c|}
\hline $\mathrm{CMC}$ & $\# 1$ & M.W. $\simeq 100,000$ & rate of ethrification $\simeq 1$ \\
\hline $\mathrm{CMC}$ & $\# 2$ & M. W. $\simeq 300,000$ & rate of ethrification $\simeq 1$ \\
\hline
\end{tabular}

$<$ natural polysaccharide $>$

\begin{tabular}{|l||c|c|c|c|c|}
\hline & glucose & mannose & rhamnose & $\begin{array}{l}\text { glucuronic } \\
\text { acid }\end{array}$ & o-acetyl \\
\hline kelzan-D & 2 & 2 & - & 1 & $20 \%$ \\
S-130 & 2 & 1 & 2 & 1 & $4 \%$ \\
S-194 & 4 & - & 1 & 1 & $10 \%$ \\
\hline
\end{tabular}




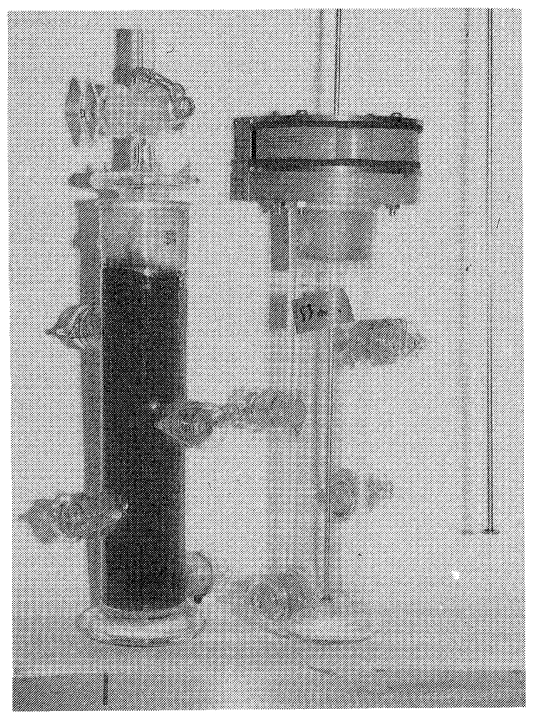

Fig. 1 Settling Column.

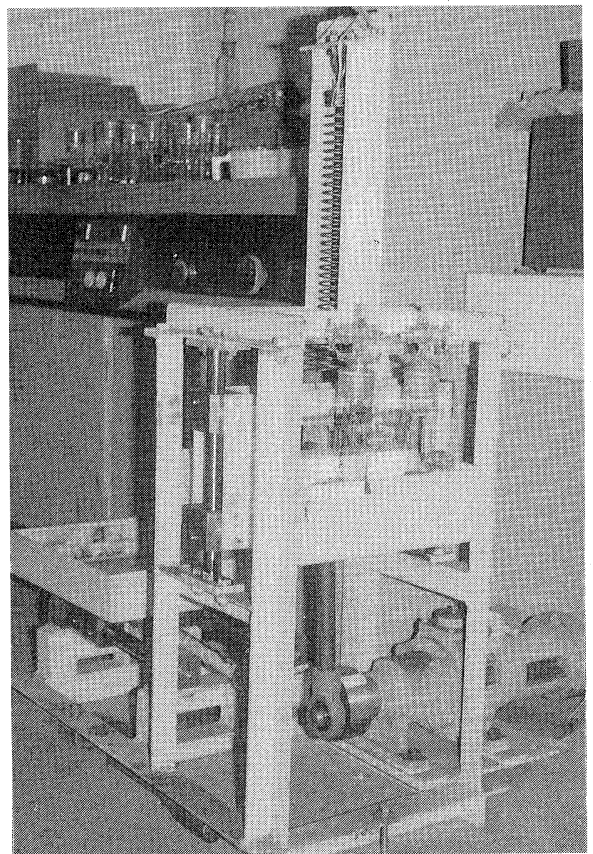

Fig. 2 Transportation simulator.

meter (IR-200, Iwamoto-seisakusho Co., LTD.). As most CWMs represent thixotropic behavior, the shear stress observed by a rheometer depends strongly on the shear history. Usui ${ }^{10}$ has proposed a phenomenological thixotropy model for CWMs in which both the equilibrium flow curve parameters and the thixotropic parameters were determined. In this study, only the equilibrium flow characteristic curves were obtained. The shear stress data were determined by applying the constant shear rate for a long time.

\section{4 Rheological measurement for stabilizing additives}

Stabilizing additives were solved into water. The solid content of additives was set at $0.1 \mathrm{wt} \%$. The equilibrium flow characteristics and elasticity of aqueous solutions were measured by means of a cone-plate rheometer (IR - 200, Iwamoto - seisakusho Co.,Ltd.).

\section{Results and Discussion}

The results of dynamic stability test for CWMs with stabilizing additives are listed in Table 2. The experimental results indicate that the addition of $0.01 \%$ kelzan-D is not effective to prevent the sedimentation of coal particles. When the concentration of kelzan-D is further increased $(0.03 \%)$, the hard pack depth decreases. However, the prise of kelzan-D is a little expensive, it is not a reasonable way to increase the concentration of kelzan-D. The addition of CMC \#1, CMC \#2 and S-130 are also ineffective. However, the addition of a small amount of S-194 $(0.01 \%)$ is effective to improve the stability of CWM.

The flow characteristic curves for the CWM with the addition of S-194 are shown in Fig. 3 . The curves for CWMs without stabilizing additives, with the addition of $0.01 \%$ kelzan-D and $0.03 \%$ 
Table 2 The results of dynamic stability tests.

\begin{tabular}{|c|c|}
\hline sample & depth of hard pack $[\mathrm{mm}]$ \\
\hline no additives & 25 \\
\hline+ kelzan-D (0.01 wt \%) & 26 \\
\hline+ kelzan-D (0.03 wt \%) & 2 \\
\hline$+\mathrm{CMC} \# 1(0.03 \mathrm{wt} \%)$ & 26 \\
\hline$+\mathrm{CMC} \# 2(0.03 \mathrm{wt} \%)$ & 10 \\
\hline+ S-130 (0.01 wt \%) & 8 \\
\hline+ S-194 $\quad(0.01 \mathrm{wt} \%)$ & 2 \\
\hline
\end{tabular}

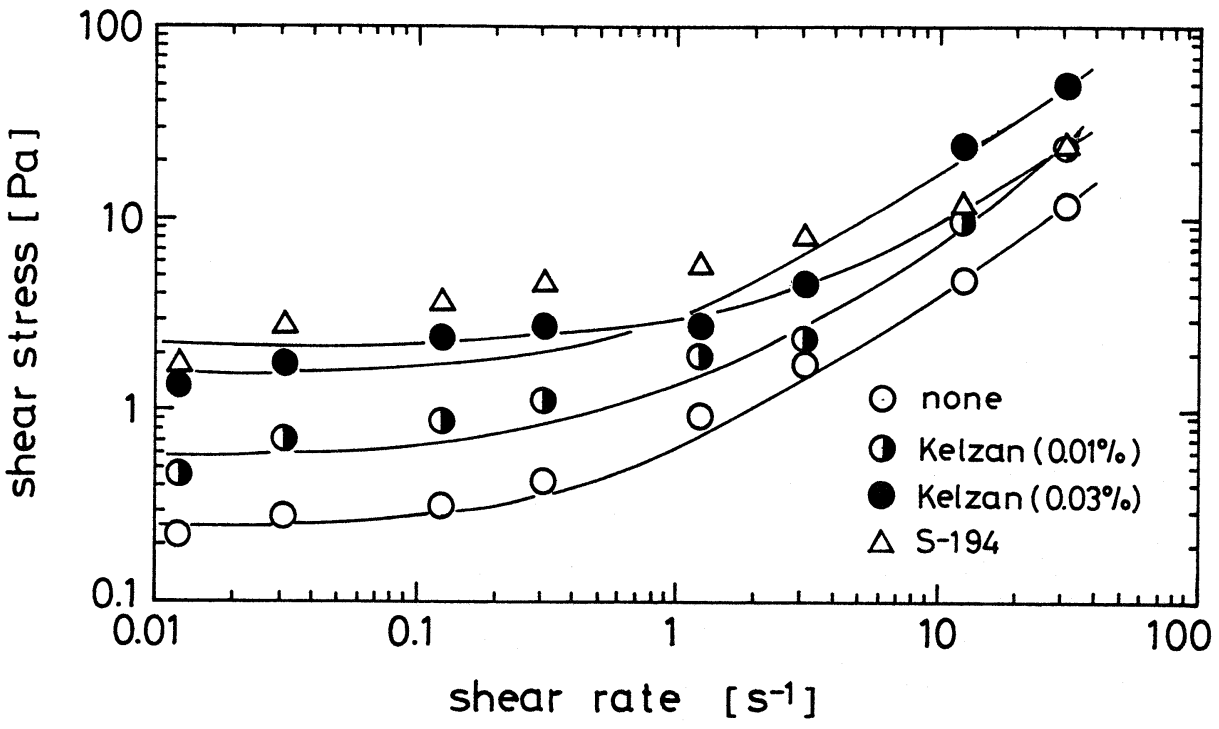

Fig. 3 The flow characteristic curves for the CWMs

kelzan-D are also shown. The ordinate represents shear stress $(\tau)$ in unit of Pa, while the abscissa indicates shear rate $(\dot{\gamma})$ in unit of $\mathrm{s}^{-1}$. The experimental results shown in Fig. 3 indicate that the yield stress of the CWM with $0.03 \%$ kelzan-D is almost the same as that of the CWM with $0.01 \%$ S-194. Furthermore, in high shear rate range, CWM with S-194 shows lower shear stress level. These results demonstrate that S-194 is effective in forming the internal structure of the CWM and dose not increase the viscosity of CWM compared to a CWM with kelzan-D.

The flow characteristic curves for aqueous solutions of CMC \#1, CMC \#2, kelzan-D and S-194 are shown in Fig. 4 (log-log) and Fig. 5 (liner-liner). The dynamic viscosity data obtained for these solutions is also shown in Fig. 6. These figures indicate that CMC solutions show the Newtonian flow properties and others show the pseudoplastic flow characteristics. In particular, it might be noticed that S-194 have a high value of yield stress. This phenomenon suggests that S-194 is effective to produce a network structure in CWMs.

Taka $^{4}$ suggested that the rheological properties of a CWM with a stabilizing additive is somewhat similar to that of aqueous soulution of the same stabilizing additive. So it is expected that 


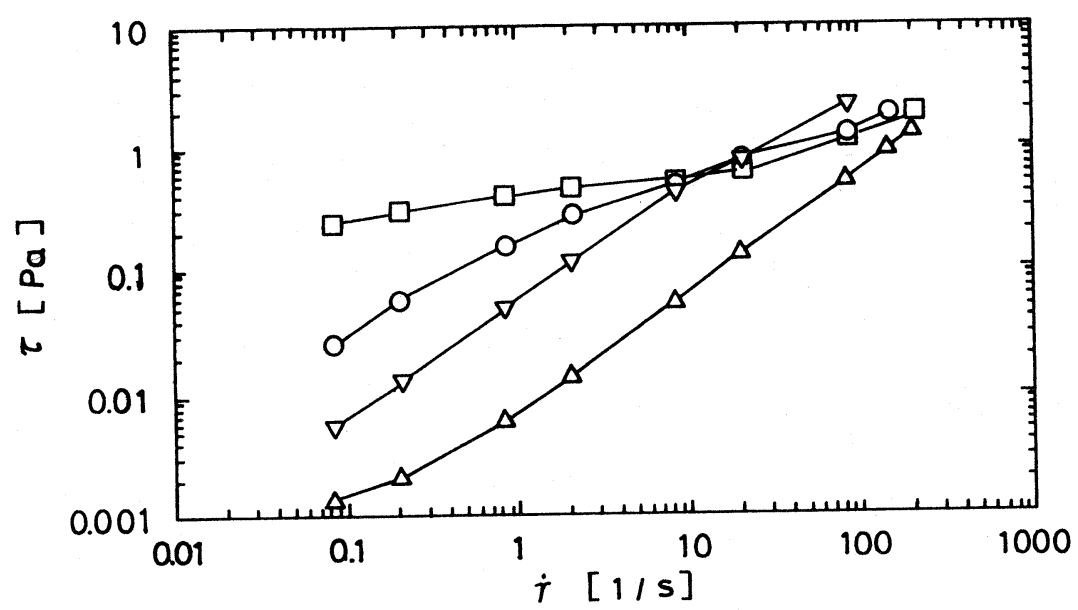

Fig. 4 The flow characteristic curves for the samples (log-log). $\triangle$; CMC \#1 $\nabla$; CMC \#2 $\bigcirc$; kelzan-D $\square$; S-194

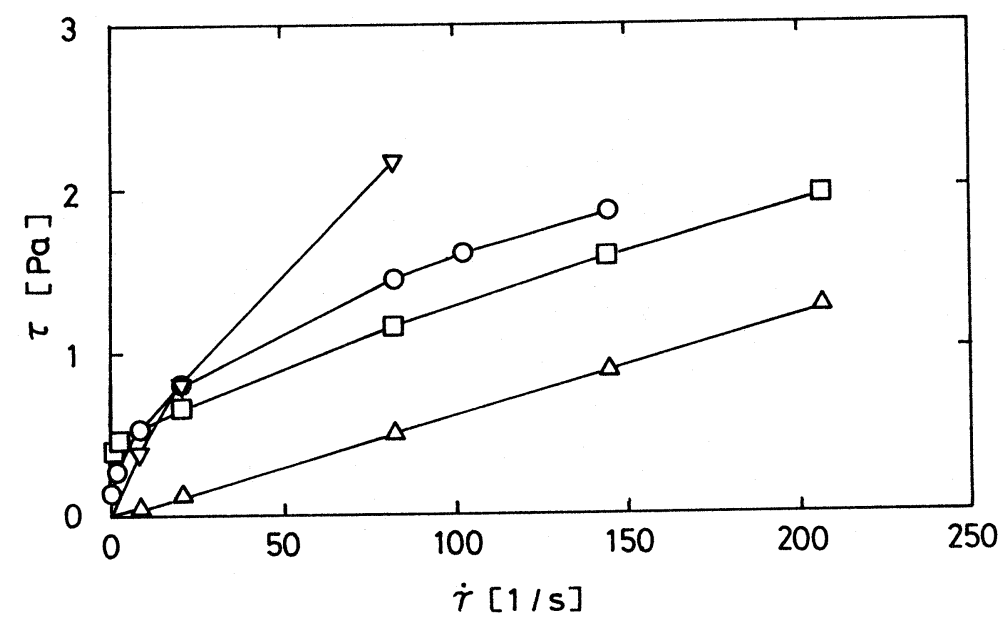

Fig. 5 The flow characteristic curves for the samples (liner-liner). $\triangle$; CMC \#1 $\nabla$; CMC \#2 $\bigcirc$; kelzan-D $\square$; S-194

rheological properties of CWMs depend greatly on the added stabilizing additives even if the concentration of stabilizing additives are very small compared to coal particles. Therefore, CWMs with the addition of S-194 are expected to build up a strong network structure which prevents the sedimentaion of coal particles. Furthermore CWM with S-194 shows a good fluidity because the viscosity decrease significantly at the high shear rate region. These characteristics may cause thixotropic properties of CWMs with S-194.

Fig. 7 shows the results of elasticity for the aqueous solutions of additives. The ordinate represents the dynamic rigidity $\left(\mathrm{G}^{\prime}\right)$ in unit $[\mathrm{Pa}]$, while the abscissa indicates frequency of the plate vibration $(\omega)$ in unit $[\mathrm{Hz}]$. It is clear from this figure and Table 2 that the stabilizing additives 


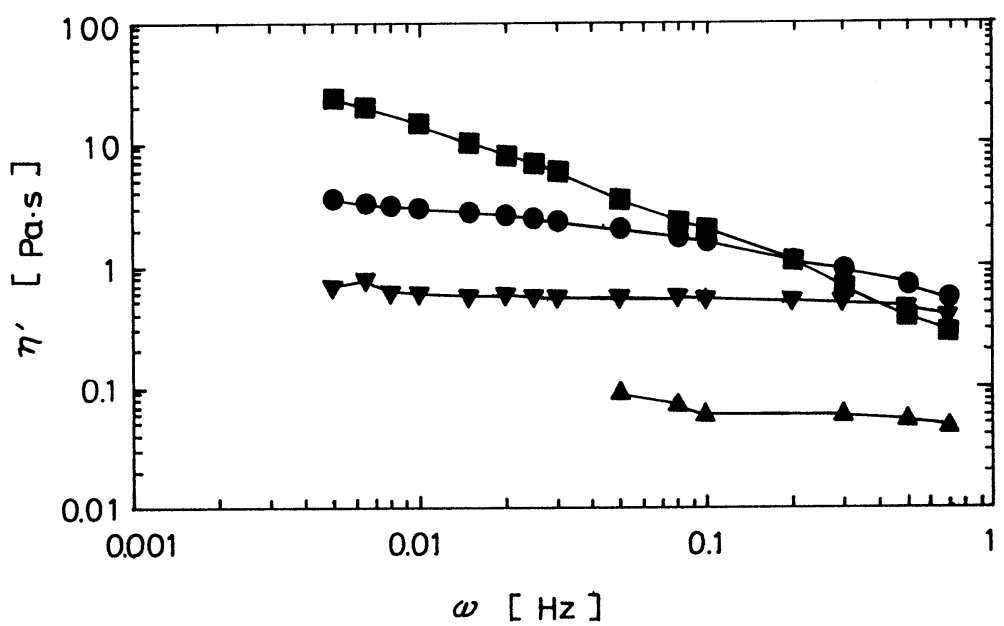

Fig. 6 Dynamic viscosity data for the samples.

$\Delta ; \mathrm{CMC} \# 1 \quad \nabla$; CMC \#2 ; kelzan-D

; S-194

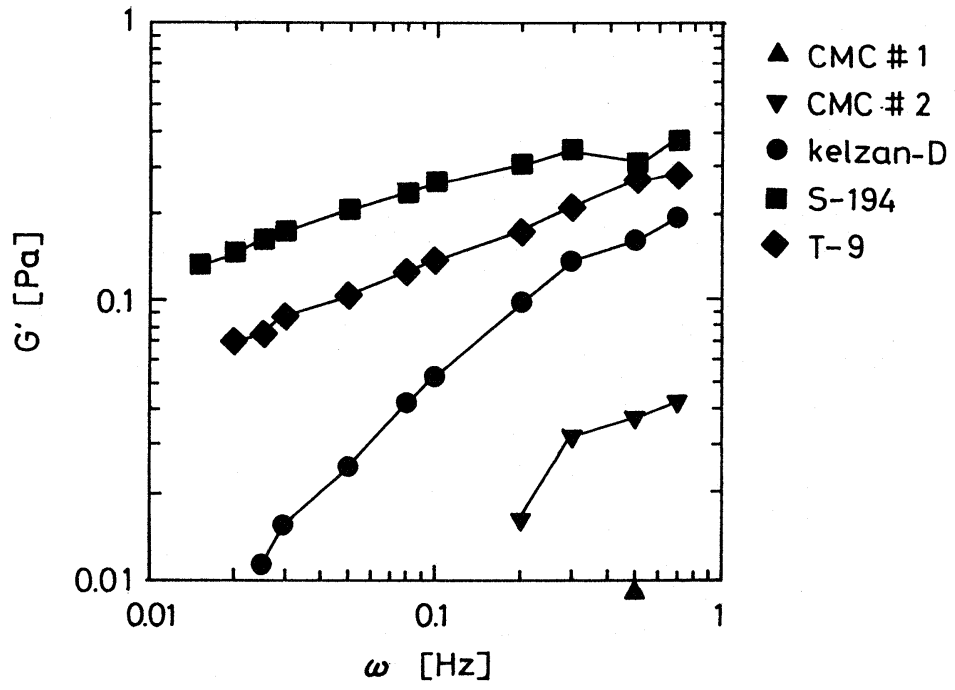

Fig. 7 The results of dynamic rigidity of the samples.
$\Delta ; \mathrm{CMC} \#$
$\nabla$; $\mathrm{CMC} \# 2$
; kelzan-D
— S-194
; T-9

of which aqueous solutions has a high value of $G^{\prime}$ contribute to improve the stability of CWMs.

Here, structures of CMC and S-194 are shown in Fig. 8. CMC consists of glucoses and has a long chain structure. Some glucoses have ionic functions, and these functions may be effective to adsorb on the coal surfaces. CMC has no ramified chains (branch). While S-194 consists of glucoses, glucuronic acids, and rhamnoses. The molecular weight of S-194 is several millions.

S-194 has many branches which may entangle with each other, and contributes to build a bridged structure in a CWM. Furthermore, S-194 also has sensible functions which are effective to adsorb on the coal surfaces. 


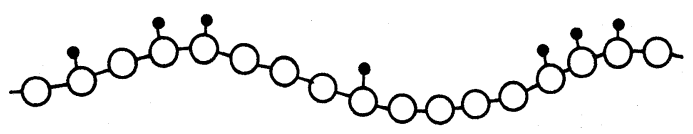

(a) $\mathrm{CMC}$
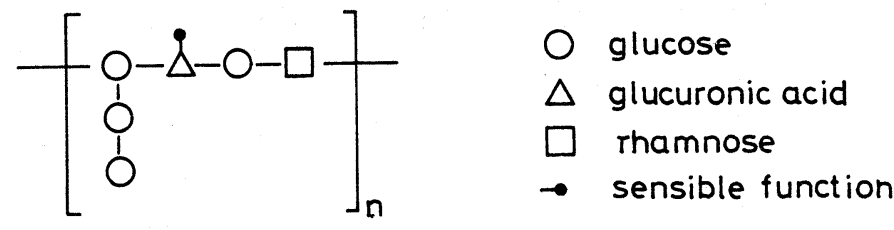

(b) $5-194$

Fig. 8 Structures of CMC and S-194.

The dynamic rigidity for aqueous solution of another polysaccaride was also measured. This stabilizing additive is named T-9 (Dainippon Pharmaceutical Co., Ltd. : “T-9” is a provisional name) and has many branches. The molecular weight of T-9 is about two millions. The value of dynamic rigidity for T-9 aqueous solution shown in Fig. 7 was larger than that of kelzan-D, so T-9 was expected to improve the stability of CWMs. However, from the results of dynamic stability test for the CWM with T-9, unnegligible amount of hard pack could be seen at the bottom of the glass cylinder.

These results indicate that the dynamic rigidity, G', represents the strength of the elasticity, but there is no information for an internal structure, e.g. degree of coagulation, dispersion, or interaction between molecules of additives and the particles, etc. On the other hand, T-9 has $-\mathrm{SO}_{3}$ functions which are not so sensible in water. The molecular chains of T-9 may not spread widely, so they could not build up a strong network structure in the CWM. It is considered that an effective stabilizing additive must have both sensible functions and branches.

\section{Conclusion}

Dynamic stability and rheological characteristics of CWMs with stabilizing additives are measured. Five kinds of stabilizing additives were tested. The experrimental results show that S-194, a kind of polysaccharides, is effective to improve the stability of CWMs. From the results of the dynamic rigidity measurement for aqueous solutions of stabilizing additives, it has become evident that the effective additives must have a high value of dynamic rigidity. It is concluded that an effective stabilizing additive must have both sensible functions and branches.

\section{Acknowledgment}

The author appreciate the experimental assistance of student colleague (S. Kobayashi).

\section{References}

1) Nakanishi,T. et al. : Proc. of 14th Int. Conf. on Coal and Slurry Technology, 321 (1989)

2) Sparks, R.W. et al. : Proc. of 4th Int. Conf.on Coal Slurry Combustion, Session V (1982)

3) Fogler, H. Scott : US DOE Rep. No. PC/80517-T4 (1986) 
4) Takao, S. et al. : Proc. 8th Int. Symp. on Coal Slurry Fuels Preparation and Utilization, 55 (1986)

5) Usui,H. et al. : J. Chem. Eng. Japan, 20, 192 (1987)

6) Colegrove, G. T. : Ind. End. Chem. Prod. Res. Dev., 22, 456 (1983)

7) Sandford, P.A. et al. : Pure \& Appl. Chem., 56, 879 (1984)

8) Francois Paul et al. : Biotech. Adv., 4, 245 (1986)

9) Usui, H. et al. : Proc. of Int. Conf. on Coal Science, F11, 983 (1989)

10) Usui, H. et al. : J. Chem. Eng. Japan, 18, 519 (1985) 